(C) 2010 ACM, Inc. Access to this work was provided by the University of Maryland, Baltimore County (UMBC) ScholarWorks@UMBC digital repository on the Maryland Shared Open Access (MD-SOAR) platform.

Please provide feedback

Please support the ScholarWorks@UMBC repository by emailing scholarworks-group@umbc.edu and telling us what having access to this work means to you and why it's important to you. Thank you. 


\title{
Maintaining Levels of Activity using a Haptic Personal Training Application
}

\author{
Huimin Qian \\ Department of Information Systems \\ UMBC \\ Baltimore, MD 21250 USA \\ huimin1@umbc.edu

\section{Ravi Kuber} \\ Department of Information Systems \\ UMBC \\ Baltimore, MD 21250 USA \\ rkuber@umbc.edu

\section{Andrew Sears} \\ Department of Information Systems \\ UMBC \\ Baltimore, MD 21250 USA \\ asears@umbc.edu
}

\begin{abstract}
This paper describes the development of a novel mobile phone-based application designed to monitor the walking habits of older adults. Haptic cues integrated within the prototype, are designed to inform an individual of changes which should be made to maintain a prescribed level of activity. A pilot study was conducted with fifteen older adults walking at varying speeds, both with and without the presence of assistive haptic feedback from the prototype. The results confirm that more steps were taken when haptic feedback was provided while walking at normal and fast paces. However, results also indicate that further refinements would be needed to improve the identification of haptic cues while individuals are in motion.
\end{abstract}

\section{Keywords}

Mobile devices, haptic vocabulary, older adults

\section{ACM Classification Keywords}

H5.2 [Information Interfaces and Presentation]:

User Interfaces- Haptic I/O

\section{General Terms}

Human Factors 


\section{Introduction}

Maintaining an appropriate level of activity is vital for older adults to improve the suppleness of joints and to reduce the occurrence of stroke and heart disease. Moderate levels of walking are recommended by Stessman et al. [1] for purposes of 'successful aging'. The reasons for older adults not performing regular exercise include low levels of motivation, the lack of available facilities, perceived excessive cost, and the lack of partners to share being active with [2].

To encourage physical exercise, it is hypothesized that touch-based cues integrated into a device which older adults already own (e.g., a mobile telephone), can assist individuals in maintaining a constant pace while walking, by indicating when they need to modify their pace. The haptic channel is an ideal method of transmitting information, reducing the need to consult the visual interface whilst in motion. Users are therefore able to concentrate on their surrounding environment (e.g. traffic surrounding the area they are walking), speak with others whilst perceiving feedback discretely, or listen to music whilst walking.

\section{Related Work}

Research has shown that visual and auditory feedback can aid physical activities. "StepMan" [3] captures information such as the pace and pulse from joggers. The speed of music played by "StepMan" is altered in real time to adjust to levels of activity, meaning that the joggers do not need to stop and select specific tracks to match their pace. "IM4Sports" [4] offers a 'pace-influencing' mode, where the tempo speeds up or slows down encouraging users to change their speed accordingly. However, the impact of haptic feedback on exercise remains largely uninvestigated. Ferber [5] studied the effectiveness of five haptic cues designed to assist users when maintaining levels of workout intensity on a stepper machine. Vibrations were integrated into the pedals, and other feedback such as resistance was provided to the legs. Results showed that the haptic signals resulted in participants stepping as much as $1.7 \%$ faster with as much as $3.95 \%$ additional power output.

Results from previous studies have also revealed the benefits of using haptic icons as a way of conveying semantic information via mobile devices $[6,7]$. These studies indicate that haptic signals could be identified with a high degree of accuracy. Our research builds on these earlier results by designing perceivable haptic icons to integrate with a commercial device already owned by many potential users: a mobile telephone. The aim was to develop a solution to provide older adults with a portable, inexpensive system to maintain a predefined level of activity.

\section{Prototype Design}

Software was designed for a Nokia N95 phone (Figure 1). The accelerometer built in the mobile phone, is used to count steps, and haptic feedback is used to convey information to the user: move faster or move slower. Unlike other solutions, our prototype uses commercially available phone-based technologies, and automatically calculates the user's pace.

\section{Counting Step Numbers}

Our software captures tri-axis acceleration data. Signal processing is performed on the raw data using a Gaussian filter. To identify the number of steps taken in a specific time period, the program calculates the number of positive wave crests. 


\section{Producing Vibration Signals}

Previous research suggests that structured vibration pulses with different durations, which occur at the same area of skin, can be composed into rhythmic units which are detectable by users [8]. Consequently, in our studies, we designed a pair of vibrations with two different rhythms to encode two messages. Both the pulse duration time and interval time between pulses were manipulated. A fixed high level of vibration intensity was used to ensure cues would be perceivable.



Figure 1: Nokia N95 (http://www.nokia.com)

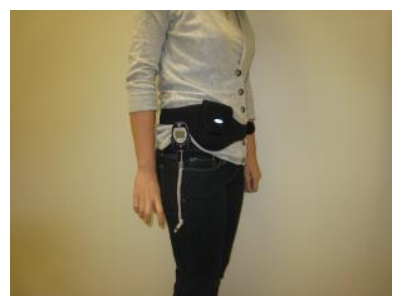

Figure 2: Handset affixed to participant's waist
In an earlier study [11], seven pairs of vibration signals were designed. To differentiate the cues, the pulse durations and intervals were manipulated. Results revealed that participants ranked the following pair of stimuli as the easiest to distinguish from each other.

- Cue 1: duration 200ms, interval 0.5s

- $\quad$ Cue 2: duration $800 \mathrm{~ms}$, interval $0.5 \mathrm{~s}$.

In subsequent experiments, these vibration cues were used to convey messages to users, as they could be differentiated more effectively when compared to the other six pairs that were studied.

\section{Experiment: Identifying the effectiveness of the application to count the number of steps taken}

Previous studies conducted by Tudor-Locke et al. [9] demonstrated the validity of using simple, inexpensive pedometers for assessing physical activity in both research and practice. To validate the step counting algorithm, the step counting results from the mobile phone were compared to the values generated by a widely used commercial pedometer (Omron HJ-112). A walking test was designed, with fifteen participants (aged 18 to 25) walking along a designated, flat, indoor path at various rates of pace. Both the mobile phone and the pedometer were attached to participants using a waist belt (Figure 2). To determine the difference between the two devices, the following calculation was used: ([N95 steps - pedometer steps]/pedometer steps) $\times 100$. Our goal was to obtain a consistent ratio between the two solutions, not necessarily to get exactly the same number of steps from both devices.

\section{Procedure}

Participants were introduced to the N95 and pedometer, and asked to walk for a period of two minutes to familiarize themselves with carrying devices using the belt. They were then asked to walk at three rates of pace: normal, slower than normal, and faster than normal. They were asked to determine these rates themselves. The order of the three rates was randomized and participants walked at each rate for two minutes, followed by an optional break. Finally, participants were asked to walk using a combination of the three rates (e.g. 90 seconds at a fast pace, 90 seconds at a slow pace, 90 seconds at their normal pace). To signal participants to start and stop walking, an auditory cue was played via the N95 device. 


\section{Results \& Discussion}

A paired sample t-test was conducted to examine the differences between step numbers counted by the two devices. The number of steps did not differ significantly between devices when walking at a normal pace $(p=0.374)$, a faster than normal pace $(p=0.893)$ and at a combination of paces $(p=0.320)$. However, in the slow walking condition, the step numbers differed significantly between devices $(p<0.005)$. Research suggests that this might be a common problem, with Cyarto's studies [10] showing that the pedometers significantly underestimated walking rates for nursing home residents when they walked slowly.

Thirteen out of the fifteen participants strongly agreed that it was comfortable to move around while wearing the phone attached to their waist. One participant commented that his right arm sometimes hit the mobile phone while walking, and the ability to position the phone himself would have been of benefit. Twelve participants indicated that they would use such a device with vibration signals in the future as an aide in maintaining their level of activity, with the other three suggesting that they would benefit from using existing pedometers. Based on a detailed analysis of these results, the algorithm was adjusted to count step numbers more accurately when walking slowly.

\section{Pilot Study: Identifying the effectiveness of haptic cues integrated into the prototype}

To study the effectiveness of haptic communications in the context of older adults maintaining a predefined pace when walking, a within-subjects experiment was designed. Participants walked at varying speeds with and without haptic cues. We recruited fifteen older adults, all aged over 60 ( 8 female and 7 male). Their average age was 69.81 (STDEV 7.02), average weight was 156.5 lbs (STDEV 21.21) and average BMI (Body Mass Index) was 23.97 (STDEV 2.00). All participants lived independently and reported that they were able to perform activities such as walking, swimming, and biking without assistance. None of the participants had taken part in the earlier study.

Table 1. Formation of Haptic Vocabulary

\begin{tabular}{|l|l|l|l|}
\hline Cue & Duration & Interval & Meaning \\
\hline Stimulus One & 200 & 0.5 & Walk Faster \\
\hline Stimulus Two & 800 & 0.5 & Walk Slower \\
\hline
\end{tabular}

The distinguishable vibration cues from our earlier study [11] were mapped to communicate the need to move faster or slower. Informal testing with users enabled us to identify that a shorter stimulus indicated to the majority to walk faster, while longer bursts indicated to move more slowly (Table 1 ).

\section{Procedure}

Participants were provided with five minutes of training perceiving vibration cues with the N95 affixed to their waists. In order to obtain participants' normal rate of speed, participants were asked to walk at a comfortable pace around an indoor space for a period of 2 minutes, with both the $\mathrm{N} 95$ and pedometer. No haptic feedback was presented during this training period. Using the resulting data, the participant's normal step rate 
denoted as $\mathrm{N}_{\mathrm{B}}$ (steps/minute) was computed. The desired fast step rate was set at $\mathrm{N}_{\mathrm{F}}=120 \% * \mathrm{~N}_{\mathrm{B}}$ (steps/minute) and desired slow step rate was $\mathrm{N}_{\mathrm{S}}=80 \% * \mathrm{~N}_{\mathrm{B}}$ (steps/minute). Next, participants were asked to walk for two minutes at each of the following rates:

- Normal Pace - Haptics: Enabled

- Faster Than Normal Pace - Haptics: Disabled

- Faster Than Normal Pace - Haptics: Enabled

- Slower Than Normal Pace - Haptics: Disabled

- Slower Than Normal Pace - Haptics: Enabled

The order of these five conditions was randomized. An auditory signal indicated when participants should start and stop walking. After each condition, the average step rate was calculated and participants' comments were recorded.

\section{Results and Discussion}

Findings revealed that when haptic cues were presented to participants to modify levels of activity, they averaged six more steps when walking at a normal pace, and three more steps were taken at a faster than normal pace. To determine the impact of vibrations to maintain levels of pace, repeated measures analysis was performed upon the ratio between the target pace and actual pace. Figure 3 presents the ratios by condition. Ratios closer to zero indicate that vibrations could have aided participants to maintain normal and slower than normal levels of pace. However, further statistical testing would be needed to verify this claim.

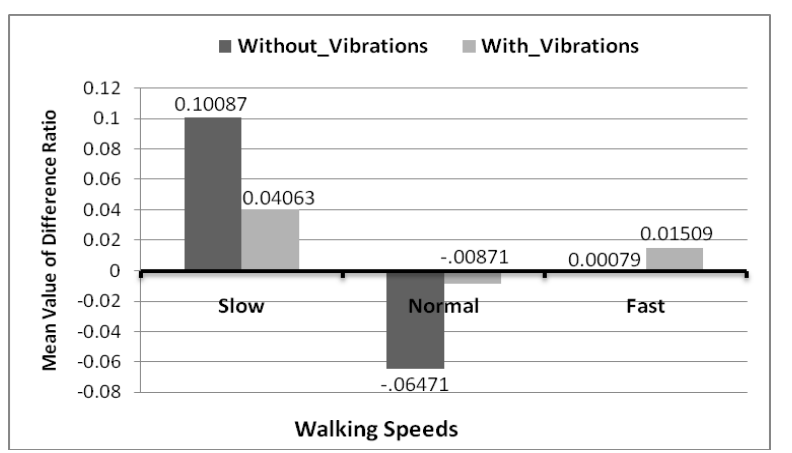

Figure 3. Ratio of step number difference ratio in self paced normal, fast and slow walking speed

Thirteen out of fifteen participants agreed that haptic cues, if designed appropriately, could be useful in helping them better maintain levels of exercise intensity, but they also suggested using more distinguishable vibration feedback in the future.

Participants were observed slowing down to wait or listen for the haptic cues, in order to process them more effectively. They suggested that feedback which was more perceivable while in motion, would be beneficial. A longer training period would also aid retention of the meanings associated with effects, as it was the first time that these participants had been introduced to such a task.

Six out of fifteen participants indicated a willingness to use a mobile phone to detect step counts in the future. The limited enthusiasm was attributed, in large part, to infrequent usage of mobile phones with just five participants using their phones for tasks other than occasionally making/receiving calls and text messaging. Some participants expressed concerns that adding such 
an application may impede their more common tasks (e.g. occlusion of feedback from incoming text messages).

\section{Conclusion and Future Work}

The results of our study showed that haptic cues can be used to modify the behavior of an individual in real time by encouraging them to take a greater or lesser number of steps. Although not statistically significantly, the smaller differences between the actual and desired walking speeds when haptic feedback was provided indicate that touch could potentially help participants adjust their overall walking speed, but our results also show that haptic cues which are more easily distinguished while walking would be useful.

In terms of future work, as the highest possible intensity of vibration was integrated into the N95 mobile phone interface, we are exploring methods of amplifying these vibrations. One possibility is the use of a low-cost attachment to a commercial mobile phone to strengthen signals. The vibration-based feedback should also be tested longitudinally to identify the challenges experienced when walking at different speeds in real-world environments (e.g. indoor and outdoor locations, flat and bumpy terrain).

\section{References}

[1] Stessman, J., Maaravi, Y., Hammerman-Rozenberg, R. and Cohen, A., The effects of physical activity on mortality in the Jerusalem 70 -Year-Olds Longitudinal Study. J Am Geriatr Soc 48: 499-504, 2000.

[2] Physical Activity Monitor. Canadian Fitness and Lifestyle Research Institute, 1995.
[3] Bieber G. and Diener H., StepMan - New kind of Music Interaction, HCII 2005, July 22-28.

[4] Wijnalda, G. Pauws, S. Vignoli, F. and Stuckenschmidt, H., A Personalized Music System for Motivation in Sport Performance, IEEE Pervasive Computing, vol. 4, no. 3, pp. 26-32, July-Sept. 2005. [5] Ferber, A.R., Affecting exercise intensity through haptic communications, Master's thesis, Northwestern University, Evanston, IL, June 2007.

[6] Brewster, S. and Brown, L. M., Tactons: structured tactile messages for non-visual information display. AUIC '04, 15-23.

[7] Brown, L. M. and Kaaresoja, T., Feel who's talking: using tactons for mobile phone alerts. CHI '06, 604-609, ACM, 2006.

[8] Gunther, E., Skinscape: A Tool for Composition in the Tactile Modality. Massachusetts Institute of Technology. Masters of Engineering, 2001.

[9] Tudor-Locke, C., Williams, J.E., Reis, J.P. and Pluto, D. Utility of Pedometers for Assessing Physical Activity: Convergent Validity. , Sports Medicine, Volume 32, Issue 12, pp 795-808, 2002.

[10] Cyarto, E.V., Myers, A.M. and Tudor-Locke, C., Pedometer accuracy in nursing home and communitydwelling older adults, Medicine \& Science in Sports \& Exercise, Volume 36 Issue 2 pp 205-209, Feb 2004.

[11] Qian, H., Kuber, R. and Sears, A., Towards Identifying Distinguishable Tactons for Use with Mobile Devices. ASSETS'09, 257-258, ACM, 2009. 